\title{
Workshop on inherited disorders and their genes in different European populations, Obernai, Strasbourg, France, 26-30 November 1993
}

\author{
(Organiser Albert de la Chapelle, Helsinki)
}

This unusual meeting, held under the auspices of the European Science Foundation, brought together around 100 people, drawn almost equally from the fields of clinical genetics, population and mathematical genetics, and molecular genetics. Its principal aims were to encourage the interaction of these groups, to see how the increasing information available on the distribution of human disease mutations might throw light on the genetic structure of European populations, and to assess the implications of such population based information for health care.

The workshop opened with some general reviews of our current knowledge of what might be considered the foundations, including the patterns of ancient migrations deduced from classical genetic markers (Robert Sokal, New York) and the influence of chance events on small island populations (Derek Roberts, Newcastle). The general approach to using molecular haplotype data and linkage disequilibrium was described by Eric Lander (Boston), while a series of contributors from Germany (Vogel, Krawczak, Grimm) dealt with different mechanisms and patterns of human mutation.

A number of specific diseases were then discussed in detail, notably three where abundant information is already available on the European distribution of mutations: cystic fibrosis, phenylketonuria, and the thalassaemias. All three disorders showed striking differences between countries, a point of clear importance to health care in relation to carrier screening and prenatal diagnosis (cystic fibrosis, $\beta$ thalassaemia) and to treatment and prognosis (PKU). It was encouraging to see how far the formation of different consortia had encouraged the collection of data even on populations that were remote or without facilities for molecular analysis. For all three diseases, extensive maps were available on mutation distribution, but the significance of these was quite a different matter.

It was at this point of the workshop that population geneticists began to question the nature of the data collected by their clinical and molecular colleagues, and in particular the validity of their sometimes sweeping conclu- sions regarding how the different genes and populations had entered and spread through Europe. Sample source and size, absence of haplotype data, and the potential effects of selection were some of the points that population geneticists rightly regarded as essential to resolve before any clear conclusions could be reached.

A second specific topic covered in some detail was the unusual frequency of genetic disorders in particular population groups, notably Finns and Jews. Both populations have been the subject of much previous work, reviewed by Reijo Norio and Arno Motulsky respectively. The effects of isolation and population fluctuation have clearly made these populations very different from the majority of European populations, with a considerable number of characteristic mendelian disorders, some dominant, most recessive, occurring at greatly increased frequency, while other widely distributed genetic disorders are rare or even absent. For these countries the practical value of a detailed knowledge of the specific mutations responsible was especially evident.

Again, however, the relationship of these rare disorders to the overall genetic structure of the populations was far from clear, and the need for caution in generalising from rare to common disorders was stressed. In the case of Jewish populations there was no agreement as to how distinct these are in overall genetic terms from their countries of previous origin.

The final specific subject was the island and other isolated populations colonised by Europeans, with their resultant genetic disorders. Réunion (populated from France) and the Azores (from Portugal) provided striking examples; the general movement of disease genes along the lines of Portuguese colonisation was described by Jorge Sequeiros, and the corresponding patterns in South Africa by Trefor Jenkins. Perhaps the most satisfying synthesis of clinical and population based approaches is provided by Quebec, where the demography and population origins from France have received as detailed study as the pattern of diseases in French-Canadians.

How far did the meeting fulfil its aims? It is always rash for someone involved in the plan- 
ning to express a view, but in terms of establishing contacts between the different groups of workers there can be no doubt that it proved extremely rewarding. Clinical and laboratory workers were given a clear awareness of how careful they must be in collecting and interpreting their data, while the theoreticians realised that new sources of data are becoming available that give scope for rigorous analysis and which can be integrated with other population data in the construction of any general hypothesis on movement and evolution of populations. Several specific collaborations have been set in motion as a result of the meeting.

Regarding the more general contribution that the study of disease genes can make to the genetic structure of European populations, many clinical and molecular geneticists probably went away less confident and more cautious than they came. Despite the striking differences in the frequency of rare disease mutations across Europe, the overall genetic structure and development of the different European populations is likely to be extremely complex, and will probably be elucidated only by a synthesis of all the data that can be collected on classical genetic and molecular (including mitochondrial) markers, in conjunction with linguistic, archaeological, and other evidence. Disease mutation data will play a role, but it will be dangerous to base general conclusions on them alone.

The third aim, of contributing to health care by establishing detailed knowledge of the distribution of mutations for different diseases, will undoubtedly have been helped by the workshop. It has become abundantly clear that such knowledge is essential for any programme of genetic diagnosis, carrier detection, or prenatal testing for a mendelian disorder, and that one cannot assume this from what is known from other populations.

A second meeting is planned for two years time, probably in Barcelona in late 1995. By then, we shall undoubtedly have not just more, but more systematically collected and analysed data on disease mutation distribution in Europe, while our understanding of how European populations have evolved should also have grown. There is at least a good chance that we may be able to begin to combine the different types of data, and to see how the differing patterns of inherited disease relate to the overall genetic structure of the complex network of populations that together make up Europe.

PETER $S$ HARPER

Institute of Medical Genetics, University of Wales College of Medicine, Heath Park, Cardiff CF4 $4 X N$, UK 